\title{
ON THE GAUSS MAPS OF SINGULAR PROJECTIVE VARIETIES
}

\author{
E. BALLICO \\ (Received 24 February 1999; revised 2 February 2001) \\ Communicated by I. Aitchison
}

\begin{abstract}
Here we study the dimension $\delta(m, X)$ of the general fibers of the $m$-Gaussian map of a singular $n$ dimensional variety $X \subset \mathbf{P}^{N}$. We show that for all integers $a, b, c, d$ with $n \leq a<b \leq c<d \leq N-1$ and $a+d=b+c$ we have $\delta(a, X)+\delta(d, X) \geq \delta(b, X)+\delta(c, X)$. If $\delta(X, N-1)$ is very large we give some classification results which extend to the singular case some results of Ein.
\end{abstract}

2000 Mathematics subject classification: primary 14N05, 14M15, 14B05.

Keywords and phrases: Gauss map, projective variety, Grassmannian, Gauss map for singular varieties, tangency, contact locus, dual variety, adjunction mapping.

\section{Introduction}

We work over an algebraically closed field $\mathbf{K}$. We are mainly interested in the case char $(\mathbf{K})=0$. Let $G(m+1, N+1)$ be the Grassmannian of all $m$-dimensional projective subspaces of $\mathbf{P}^{N}$. Let $X \subset \mathbf{P}^{N}$ be an integral variety with $\operatorname{dim}(X)=n$. For every integer $m$ with $n \leq m \leq N-1$ the $m$-Gauss map $\gamma_{m, X}$ of $X$ and the $m$-defect $\delta(m, X)$ of $X$ are defined in the following way. Set $A^{\prime}(m, X):=\{(P, L) \in$ $\left.X_{\text {reg }} \times G(m+1, N+1): T_{P} X \subseteq L\right\}$ and let $A(m, X)$ be the closure of $A(m, X)$ in $X \times G(m+1, N+1) \subset \mathbf{P}^{N} \times G(m+1, N+1)$. Let $\pi_{1}: X \times G(m+1, N+1) \rightarrow X$ and $\pi_{2}: X \times G(m+1, N+1) \rightarrow G(m+1, N+1)$ be the projection on the first (respectively second) factor. Set $\gamma_{m, X}:=\pi_{2} \mid A(m, X)$ and call $\gamma_{m, X}(X)=\pi_{2}(A(m, X))$ the $m$ Gauss image of $X$. Let $\delta(m, X)$ be the dimension of the general fiber of $\gamma_{m, X}$, that is, the dimension of the contact locus of a general $m$-dimensional linear subspace of $\mathbf{P}^{N}$ which is tangent to $X$ at some smooth point. In particular, $\gamma_{N-1, X}(X) \subset \mathbf{P}^{N *}$ is the dual variety $X^{*}$ of $X$ and (with the identification of $A^{\prime}(n, X)$ with $X_{\text {reg }}$ ) the morphism

The author was partially supported by MURST (Italy).

(C) 2002 Australian Mathematical Society 0263-6115/2002 \$A2.00+0.00 
$\gamma_{m, X} \mid X_{\text {reg }}$ is the usual Gauss map from $X_{\text {reg }}$ to $G(m+1, N+1)$. For the background on these topics, see [16] or [13].

In Section 2 we study the finite sequence of all $\delta(m, X), n \leq m \leq N-1$, and prove that for all integers $a, b, c, d$ with $n \leq a<b \leq c<d \leq N-1$ and $a+d=b+c$ we have $\delta(a, X)+\delta(d, X) \geq \delta(b, X)+\delta(c, X)$ (Remark 2) and that if $\delta(m, X)>\delta(n, X)$, then $\delta(m, X)>\delta(m-1, X)$ (Proposition 2). We give examples which, in our opinion, show that not much more is true. Furthermore, we study the relations between the Gauss defects of $X$ and the Gauss defects of the intersection of $X$ with a general hypersurface of degree $d$. In Section 3 we extend some of the results of [3] and [2] to the singular case; roughly speaking, we have a full extension if $\operatorname{dim}(\operatorname{Sing}(X))=0$ (excluding cones). In the last section we discuss not the dimension of a general fiber of a Gaussian map, but the existence of positive dimensional fibers of the ordinary Gauss map $\gamma_{n, X}$. Of course, by Zak's Tangency Theorem ([16, Theorem 1.7]) the variety $X$ cannot be smooth.

\section{The sequence of Gauss defects}

In this section we study the sequence $\delta(m, X), n \leq m \leq N-1$, of all Gauss defects of an integral $n$-dimensional variety $X \subset \mathbf{P}^{N}$. Just by the definition of the Gauss defects we have $\delta(m, X) \leq \delta(m+1, X)$ for every $X$ and every integer $m$ with $n \leq m \leq N-2$. Something more can be said (see Remark 2, Proposition 2 and Example 1), but in our opinion, not much more even for smooth manifolds (see Example 1, Example 2 and Example 3), without making some very strong restrictions on the geometry of $X$. Then we consider the relations between the Gauss defects of $X$ and the Gauss defects of the intersection of $X$ with a general hypersurface of degree $d \geq 1$.

LEMMA 1. Let $n, s$ and $N$ be fixed integers with $n<s<N$. Let $X \subset \mathbf{P}^{N}$ be an integral $n$-dimensional variety and let $Z \subset \mathbf{P}^{s}$ be a general projection of $X . X$ is reflexive if and only if $Z$ is reflexive.

Proof. Use the Hessian criterion for reflexivity ([11, Theorem 12], or [6, Theorem 3.2 and Corollary 3.3]).

REMARK 1. If $X$ is reflexive (and in particular if $\operatorname{char}(\mathbf{K})=0$ ), then for any $n$ dimensional $X$ and any $m$ the general fiber of $\gamma_{m, X}$ is a linear space (see [11, page 173] for the case $m=N-1$ and reduce to this case taking a general projection into $\mathbf{P}^{m+1}$; the general projection of a reflexive $X$ into $\mathbf{P}^{m+1}$ is reflexive by Lemma 1); alternatively, if $\operatorname{char}(\mathbf{K})=0$, see $[13,5.2$ (ii)]. 
PROPOSITION 1. Let $X \subset \mathbf{P}^{N}$ be an integral $n$-dimensional reflexive variety. Then for all integers $m$ with $n \leq m \leq N-3$ we have

$$
\delta(m+2, X)+\delta(m, X) \geq 2 \delta(m+1, X) .
$$

PROOF. Fix a general fiber $F$ of $\gamma_{m, X}$ and two general fibers $F^{\prime}, F^{\prime \prime}$ of $\gamma_{m+1, X}$ with $F \subset F^{\prime}$ and $F \subset F^{\prime \prime}$. Thus for dimensional reasons we have $F=F^{\prime} \cap F^{\prime \prime}$. Set $G:=\left\langle F^{\prime} \cup F^{\prime \prime}\right\rangle$. Hence $G$ (respectively $F^{\prime}$ and $F^{\prime \prime}$ ) is a sufficiently general fiber of $\gamma_{m+2, X}$ (respectively $\gamma_{m+1, X}$ ). Let $T\left(T^{\prime}, T^{\prime \prime}, R\right.$ respectively) be the contact locus of $F\left(F^{\prime}, F^{\prime \prime}, G\right.$ respectively). By the reflexivity of $X$ the sets $T, T^{\prime}, T^{\prime \prime}$ and $R$ are linear spaces with dimension respectively $\delta(m+2, X), \delta(m+1, X), \delta(m+1, X)$ and $\delta(m, X)$ (Remark 1). Since $T=T^{\prime} \cap T^{\prime \prime}$ and $T^{\prime} \cup T^{\prime \prime} \subseteq R$, we conclude the proof.

REMARK 2. Proposition 1 is a convexity result. Indeed, it implies that for all integers $a, b, c$ and $d$ with $n \leq a<b \leq c<d \leq N-1$ and $a+d=b+c$ we have

$$
\delta(a, X)+\delta(d, X) \geq \delta(b, X)+\delta(c, X) .
$$

PROPOSITION 2. For fixed integers $n, m$ and $N$ with $0<n<m<N$, let $X$ be an integral n-dimensional subvariety of $\mathbf{P}^{N}$ with $\delta(m, X)>\delta(n, X)$. Then $\delta(m, X)>$ $\delta(m-1, X)$.

Proof. Fix a general $P \in X_{\text {reg }}$ and a general $m$-dimensional linear space $M$ with $\operatorname{dim}(M)=m$ and $T_{P} X \subset M$. Let $C$ (respectively $C^{\prime}$ ) be the contact locus of $M$ (respectively $\left.T_{P} X\right)$. Since $\operatorname{dim}(C)=\delta(m, X)>\delta(n, X)=\operatorname{dim}\left(C^{\prime}\right)$, there is a hyperplane $A$ of $M$ containing $T_{P} X$ but no irreducible component of $C$, that is, with contact locus, $C^{\prime \prime}$, of dimension at most $\operatorname{dim}(C)-1$. Hence we conclude the proof by the generality of $P, M$ and $A$.

The following example shows that in general in the statement of Proposition 2 we cannot replace the assumption $\delta(m, X)>\delta(n, X)$ with the assumption $\delta(m, X)>0$.

EXAMPLE 1. Let $X \subset \mathbf{P}^{N}$ be the $n$-dimensional cone over an $(n-1)$-dimensional variety $Y \subset \mathbf{P}^{N-1}$ with $\delta(n, Y)=0$. Then $\delta(n+1, X)=\delta(n, X)=1$.

PROPOSITION 3. Let $X$ be an integral $n$-dimensional reflexive subvariety of $\mathbf{P}^{N}$ with $\operatorname{Sing}(X)$ finite. $X$ is a cone if and only if $\delta(n, X)>0$.

Proof. If $X$ is a cone we have $\delta(n, X)>0$. Assume $\delta(n, X)>0$. Since $X$ is reflexive, the general contact locus of an $n$-dimensional tangent space is a positive dimensional linear subspace (Remark 1 ). Since $\operatorname{Sing}(X)$ is finite, either $X$ is a cone 
with $\operatorname{Sing}(X)$ as a vertex or the general contact locus is contained in $X_{\text {reg. }}$. The latter case is excluded by Zak's Theorem of Tangency ([16, Theorem 1.7]).

In our opinion the following two examples show that if we do not make some restrictive assumptions the finite sequence of all Gauss defects of a smooth manifold does not satisfy restrictions much stronger that the ones given by Remark 2 and Proposition 2. However, since smooth manifolds with 'bad' Gauss maps are very particular, it should be possible to make reasonable assumptions and obtain better results. For instance, if $3 n \leq 2 N$ all smooth $n$-dimensional manifolds $X \subset \mathbf{P}^{N}$ with $\operatorname{dim}(X)=\operatorname{dim}\left(X^{*}\right)$ are classified $([3$, Theorem 4.5]).

EXAMPLE 2. Let $X \subset \mathbf{P}^{N}$ be a smooth $n$-fold which is a $\mathbf{P}^{n-1}$-bundle over a smooth curve $C$. Call $\pi: X \rightarrow C$ the projection. For every $P \in X$, we have $\pi^{-1}(\pi(P)) \cong \mathrm{P}^{n-1}$ and $\pi^{-1}(\pi(P)) \subset T_{P} X$. If $X$ is reflexive, then $\delta(N-1, X)=n-2$ ([10, page 360]). For the reverse when $X$ is reflexive, see [2, Theorem 3.1], or, if $\operatorname{char}(\mathbf{K})=0$, [3, Theorem 3.2]. Furthermore, for general $P \in X$ and $H, R \in X^{*}$ with $T_{P} X \subset H \cap R$, the contact loci, $L_{H}$ and $L_{R}$, of $H$ and $R$ are hyperplanes of $\pi^{-1}(\pi(P))$. Since $L_{H} \cap L_{R}$ is the contact locus of $H \cap R$, we obtain that either $\delta(N-2, X)=\delta(N-1, X)$ or $\delta(N-2, X) \equiv \delta(N-1, X)-1$. By Lemma 2 we must have $\delta(N-2, X)=\delta(N-1, X)-1$. Hence, using Proposition 1, we obtain $\delta(m, X)=\max \{0, n-1-N+m\}$ for every integer $m \geq 0$.

The following example shows a trivial way to obtain singular non-normal varieties, $X$, which have large $\delta(N-1, X)$.

EXAMPLE 3. Let $Y \subset \mathbf{P}^{N+1}$ be a smooth $\mathbf{P}^{m-1}$-bundle over a smooth curve (Example 2). Fix general $P^{\prime}, P^{\prime \prime} \in Y$ and take a general point $P$ on the line $\left\langle P^{\prime}, P^{\prime \prime}\right\rangle$ spanned by $P^{\prime}$ and $P^{\prime \prime}$. Let $f: \mathbf{P}^{N+1} \backslash\{P\} \rightarrow \mathbf{P}^{N}$ be the projection from $P$. Set $X:=f(Y)$. Since $\left\langle P^{\prime}, P^{\prime \prime}\right\rangle \cap Y=\left\{P^{\prime}, P^{\prime \prime}\right\}$ for large $N, Y$ is the normalization of $X$. By construction $X$ has a non-normal point $f\left(P^{\prime}\right)=f\left(P^{\prime \prime}\right)$ and for large $N$ (for fixed $n$ ) this is usually the only singular point of $X$. If $Y$ is reflexive, then $X$ is reflexive. Hence by [10, page 360], we have $\delta(N-1, X) \geq n-2$ and (except again trivial cases like $N=3$ and $Y$ a smooth quadric surface) we have $\delta(N-1, X)=n-2$. But we have similar 'non-normal scrolls', $X^{\prime}$, whose normalization $g: Y^{\prime} \rightarrow X^{\prime}$ has $h^{0}\left(X^{\prime}, g^{*}\left(\mathscr{O}_{Y^{\prime}}(1)\right)\right)=N+1$, that is, the map $Y^{\prime} \rightarrow \mathbf{P}^{N}$ corresponds to a complete linear system; even in these cases [10, page 360], shows that $\delta\left(Y^{\prime}, N-1\right) \geq n-2$ if $Y^{\prime}$ is reflexive. More generally, if the $n$-dimensional reflexive variety $Z \subset \mathbf{P}^{N}$ is uniruled by $t$-dimensional linear spaces we have $\delta(N-1, Z) \geq t-1$. If $W=\left\{f\left(x_{0}, \ldots, x_{N}\right)=0\right\}$ is a hypersurface of $\mathbf{P}^{N}$, there is a necessary and sufficient condition (at least if $\operatorname{char}(\mathbf{K})=0)$ to have $\delta(N-1, W)>0$ : a certain projective Hessian matrix must be divisible by $f$ (for the case $N=3$ this is an old theorem of Schläfli, see [5, 
page 23]). Hence there are non-normal reflexive surfaces $W \subset \mathbf{P}^{3}$ uniruled by lines with $\delta(2, W)>0$.

Example 2 shows why in the statements of Proposition 7 and Proposition 8 we only classified the normalization of $X$ and why in the statement of Theorem 1 we have to assume that $X$ is normal.

Now we study the relation between the Gauss defects of $X$ and the Gauss defects of the intersection of $X$ with a general hypersurface of degree $d \geq 1$.

LEMMA 2. Fix integers $n, m$ and $N$ with $2 \leq n \leq m<N$. Let $X \subset \mathbf{P}^{N}$ be an integral n-dimensional variety and $Y$ a general hyperplane section of $X$. Then $\delta(m-1, Y) \geq \delta(m, X)-1$.

PROOF. We may assume $\delta(m, X) \geq 2$. Fix a general $P \in X$ and a general $m$ dimensional linear subspace $M$ of $\mathbf{P}^{N}$ with $T_{P} X \subset M$. Let $C$ be the contact locus of $M$. Take a general hyperplane $H$ containing $P$ and set $Y:=X \cap H$. By the generality of $P, Y$ is a general hyperplane section of $X$. By the generality of $H$, we have $\operatorname{dim}(M \cap H)=m-1$ and $\operatorname{dim}(C \cap H)=\operatorname{dim}(C)-1$. Furthermore, fixing $H$ and varying $P$ in $Y$ we see that $P$ may be considered a general point of $Y$. Since $C \cap H$ is contained in the contact locus of $M \cap H$ with $Y$, we obtain the lemma.

REMARK 3. Assume $\operatorname{char}(\mathbf{K}) \neq 2$. Let $X \subset \mathbf{P}^{N}$ be an integral variety with $\operatorname{dim}(X) \geq 2$. For a general hyperplane $H$ of $\mathbf{P}^{N}$, we have $\delta(N-2, X \cap H)=$ $\max \{0, \delta(N-1, X)-1\}([6$, Theorem 5.9]).

Proposition 4. Fix integers $n, m$ and $N$ with $2 \leq n \leq m<N$. Let $X \subset \mathbf{P}^{N}$ be an integral $n$-dimensional variety and $Y$ a general hyperplane section of $X$. Then

$$
\delta(m-1, Y)=\max \{\delta(m, X)-1,0\} .
$$

PROOF. The case $m=N-1$ of the result was proved in [6, 5.9 and 5.12]. Use Lemma 1 to reduce the general statement to the case $m=N-1$.

REMARK 4. Fix integers $d, n$ and $N$ with $2 \leq n<N$ and $d \geq 2$. Let $X \subset \mathbf{P}^{N}$ be an integral $n$-dimensional variety and $Y$ the intersection of $X$ with a general hypersurface of degree $d$. By [6, Theorem 5.6], if either $\operatorname{char}(\mathbf{K}) \neq 2$ or $n$ is even, then $Y$ is reflexive and $\delta(N-1, Y)=0$. It is easy to check directly that $\delta(N-1, Y)=0$ even in the remaining case char $(\mathbf{K})=2$ and $n$ odd using a Bertini type argument. Hence for every integer $m$ with $n \leq m<N$ we have $\delta(m, Y)=0$.

In positive characteristic it is natural to give criteria for the separability of a Gauss map $\gamma_{m, X}$. In the case $m=N-1$ this is exactly the reflexivity of $X$ (see [10, 
Theorem 4]). There are at least two natural definitions for the inseparability degree of Gaussian maps ([12, page 2]); for their coincidence if $X$ is a curve, see [9]; for a discussion of the general case, see [12] and the last part of the introduction of [9].

Using Lemma 1 for $s=m+1$ and the corresponding result for $\gamma_{N-1, X}$ ([11, Theorem 4]) we obtain the following result.

PROPOSTTION 5. Fix integers $n, m$ and $N$ with $n \leq m<N$. Let $X \subset \mathbf{P}^{N}$ be an integral n-dimensional variety. The map $\gamma_{m, X}$ is separable if and only if $\gamma_{N-1, X}$ is separable, that is, if and only if $X$ is reflexive.

\section{Adjunction theory and Ein's papers}

In this section we will try to follow as closely as possible [3] and [2] to obtain results on a singular variety with $\delta(N-1, X)$ large. Roughly speaking, we are able to handle isolated singularities, while in the general case we need to add an additive factor $\operatorname{dim}(\operatorname{Sing}(X))$ to the assumptions of every statement. If $A$ is a closed subvariety of the variety $B$, let $N_{A / B}$ be the normal sheaf of $A$ in $B$.

REMARK 5. Let $X \subset \mathbf{P}^{N}$ be an $n$-dimensional integral reflexive variety with $\delta(N-1, X)>0$. Let $L_{H}$ be the contact locus of a general $H \in X^{*}$. Since $X$ is reflexive, $L_{H}$ is a linear space of dimension $\delta(N-1, X)$ ([11, page 173]). Hence if $\operatorname{Sing}(X)$ is finite, then either $L_{H} \subset X_{\text {reg }}$ or $X$ is a cone.

DEFINITION 1. Let $X$ be an integral $n$-dimensional variety, $n \geq 2$, with $\delta(N-1$, $X)>0$. Let $\Pi$ be the covering family of all $\delta(N-1, X)$-dimensional linear spaces which are limits of the family of all 'general' $\delta(N-1, X)$-dimensional contact loci. Since $X$ is closed in $\mathbf{P}^{N}$, every $R \in \Pi$ is contained in $X$. Since $X^{*}$ is irreducible, $\Pi$ is irreducible. We will say that $X$ satisfies Condition (\$) if for a general $R \in \Pi$ we have $R \subset X_{\text {reg }}$. Let $\mathbf{T}$ be the covering family of lines obtained as closure of all lines in the contact loci of general $M \in X^{*}$, that is, let $\mathbf{T}$ be the set of all lines contained in some $R \in \Pi$. We will say that $X$ satisfies Condition $(f)$ if for a general $D \in \mathrm{T}$ we have $D \subset X_{\text {reg. }}$.

By Remark 5 Condition (\$) is satisfied if $\operatorname{Sing}(X)$ is finite and $X$ is not a cone. Obviously, Condition (\$) implies Condition ( $($ ).

REMARK 6. Let $X \subset \mathbf{P}^{N}$ be an $n$-dimensional integral reflexive variety with $\delta(N-$ $1, X)>0$. We assume $\operatorname{char}(\mathbf{K}) \neq 2$. Here we assume that the contact locus, $L$, of a general $H \in X^{*}$ is contained in $X_{\text {reg. }}$. We will follow quite closely [3] and [2] and obtain some informations on $X$. In [3] and [2] the variety $X$ was assumed to 
be smooth. Since $X$ is reflexive, $L$ is a linear space of dimension $\delta(N-1, X)([11$, page 173]). Set $k:=\delta(N-1, X)$. Since $L \subset X_{\text {reg }}$, the normal sheaf $N_{L / X}$ is locally free of rank $n-k$. Since $L \subset X_{\text {reg }}, X$ is reflexive and $\operatorname{char}(\mathbf{K}) \neq 2$, the proof of [3, Theorem 2.1] works verbatim and gives [3, Theorem 2.2], that is, $N_{L / X} \cong N_{L / X}^{*}(1)$. The proof of [3, Theorem 2.3] works verbatim and gives that for every line $T \subseteq X$ we have

$$
N_{L / X} \mid T \cong \mathscr{O}_{T}^{\oplus(n-k) / 2} \oplus \mathscr{O}_{T}(1)^{\oplus(n-k) / 2},
$$

in particular, $N_{L / X}$ is a uniform vector bundle and if $\operatorname{rank}\left(N_{L} / X\right)<\operatorname{dim}(L)$, that is, if $n<2 k$, then

$$
N_{L / X} \cong \mathscr{O}_{L}^{\oplus(n-k) / 2} \oplus \mathscr{O}_{L}(1)^{\oplus(n-k) / 2}
$$

by a theorem of Tango on uniform vector bundles on $\mathbf{P}^{k}$ valid in arbitrary characteristic ([14] and [15]). As in [3, Theorem 2.3, part (b)] we obtain the existence of an irreducible family of dimension $(n+k-2) / 2$ of lines on $X$ and that for any $P \in T$ there is a family of dimension $(n+k-2) / 2$ of lines in $X$ through $P$. Using the extension of [3, Theorem 2.3], we obtain Landman's parity criterion given in [3, Theorem 2.4], that is, we obtain that $n-\delta(N-1, X)$ is even (of course, only under the assumption $L \subset X_{\text {reg }}$ ). For the same reason we have parts (a), (b), (c) and (d) of [3, Theorem 2.4], but not (a priori) part (e) of [3, Theorem 4], because of $\operatorname{dim} \operatorname{Sing}(X)$ we cannot apply the part of Lefschetz theorem stating that if $2 n>N+2$, then $\operatorname{Pic}(X)$ is generated by the hyperplane class.

PRoposition 6. Assume char $(\mathbf{K}) \neq 2$. Let $X \subset \mathbf{P}^{N}$ be a two-dimensional integral reflexive variety with $\delta(N-1, X)>0$ and $\operatorname{Sing}(X)$ finite and not empty. Then $X$ is a cone.

Proof. We have $0<\delta(N-1, X)<2$. If the contact locus of a general $H \in X^{*}$ intersects $\operatorname{Sing}(X), X$ is a cone by Remark 5. If the contact locus of a general $H \in X^{*}$ does not intersect $\operatorname{Sing}(X)$, then $2-\delta(N-1, X)$ is even by Landman's parity criterion extended in Remark 5, contradiction.

Proposition 7. Assume $\operatorname{char}(\mathbf{K}) \neq 2$. Let $X \subset \mathbf{P}^{N}, N \geq 5$, be a reflexive integral variety with $\operatorname{dim}(X)=3$, Sing $(X)$ finite and $\delta(N-1, X)>0$. Let $g: Z \rightarrow X$ be the normalization. Then either $X$ is a cone or $Z$ is a smooth scroll, say $\pi: Z \rightarrow C$, over a smooth curve $C$ and $g$ sends every fiber of $\pi$ isomorphically onto a plane contained in $X$.

Proof. By $[2$, Corollary 3.2 (a)] we may assume $\operatorname{Sing}(X) \neq \emptyset$. Since $X$ is reflexive, the generic contact locus is a linear space. Assume that $X$ is not a cone. Hence there is an irreducible covering family $\mathbf{T}$ of lines such that for a general $L \in \mathbf{T}$ we have $L \subset X_{\text {reg }}, L$ is a contact locus and $N_{L / X} \cong \mathscr{O}_{L}(1) \oplus \mathscr{O}$. Fix a general $L \in \mathrm{T}$ 
and a general $P \in L$. Hence $P$ may be considered as a general point of $X$. Since $X$ is not a cone and $\operatorname{Sing}(X)$ is finite, no line containing $P$ intersects $\operatorname{Sing}(X)$. Hence by the deformation theory there is an irreducible one-dimensional family $\mathbf{T}(P) \subset \mathbf{T}$ formed by the lines containing $P$. Since $h^{1}\left(L, N_{L / X}(-P)\right)=0, \mathbf{T}(P)$ is smooth at $L$. Set $F(P):=\{Q \in X: Q \in D$ for some $D \in \mathbf{T}(P)\}$. Since two distinct lines through $P$ intersect only at $P, F(P)$ is a two-dimensional subvariety of $X$. We have $F(P) \cap \operatorname{Sing}(X)=\emptyset$ for general $P . F(P)$ is a cone with vertex $P$ and hence $T_{P} X$ contains $F(P)$.

First assume that for general $P$ the variety $F(P)$ is not a plane. Let $R \subset \mathbf{P}^{N}$ be a general hyperplane containing $P$. Set $Y:=X \cap R$. Since $P$ is general, $Y$ may be considered as a general hyperplane section of $X$. Thus $\delta(N-2, Y)=$ $\delta(N-1, X)-1=0($ Remark 3$)$. Hence $T_{P} Y \cap Y$ has $P$ as a unique singular point and this singularity is an ordinary quadratic singularity ([6, Theorem 3.5$]$, or [11, Theorem 17, page 179]). Thus $R \cap F(P)$ has at most two irreducible components, that is, $F(P)$ is a quadratic cone. Since $N \geq 5$ and $F(P) \subset T_{P} X$, for a general $H \in X^{*}$ with $T_{P} X \subset H$ the scheme $H \cap X$ contains at least two irreducible components, one of them (that is, $F(P)$ ) being singular. Hence $Y \cap H=X \cap H \cap R$ does not have an ordinary quadratic singularity as a unique singular point, contradiction.

Now assume that $F(P)$ is a plane. Since $F(P) \cap F\left(P^{\prime}\right)=\emptyset$ for general $P, P^{\prime} \in X$, we have $N_{F(P) / X} \cong \mathscr{O}_{F(P}$. Since the Grassmannian $G(2, N+1)$ is complete, for every $Q \in \operatorname{Sing}(X)$, there is at least a plane $V(Q) \in \mathbf{T}$ with $Q \in V(Q)$. Fix any such $V(Q)$ and a plane $F \in \mathbf{T}$ with $F \cap \operatorname{Sing}(X)=\emptyset$. First assume $F \cap V(Q) \neq \emptyset$. Since $F \cap \operatorname{Sing}(X)=\emptyset$ and both $F$ and $V(Q)$ are planes, $D:=F \cap V(Q)$ is a line. For every $P \in D$, we have $F \cup V(Q) \subset T_{P} X$. Hence $T_{P} X$ is the 3-dimensional linear space $M$ spanned by $F \cup V(Q)$, that is, $M$ is tangent along $\mathrm{D}$ to $X$. Since $D \subset F \subset X_{\text {reg }}$, this contradicts Zak's Tangency Theorem ([16, Theorem 1.7]). Hence $V(Q) \cap F=\emptyset$ for every $F \in \mathbf{T}$ with $F \cap \operatorname{Sing}(X)=\emptyset$. For the same reason, if $Q^{\prime} \in \operatorname{Sing}(X)$ and $Q^{\prime} \notin V(Q)$, then $V(Q) \cap V\left(Q^{\prime}\right)=\emptyset$ for any $V\left(Q^{\prime}\right)$ with $Q \notin V\left(Q^{\prime}\right)$. By construction we have a smooth affine curve $A$ parametrizing an open subset of planes in $X$, an open subset $\Omega$ of $X_{\text {reg }}$ and a morphism $\pi^{\prime}: \Omega \rightarrow A$ with fibers as planes of $T$ not intersecting $\operatorname{Sing}(X)$. Fix $B \in X_{\text {reg. }}$. For a general hyperplane $R$ through $B$, the surface $X \cap R$ is smooth and it is a $\mathbf{P}^{1}$-bundle $\pi_{R}: X \cap R \rightarrow C_{R}$ over a smooth curve $C_{R}$. If $\operatorname{char}(\mathbf{K})=0$ and $Z$ is assumed to have only locally complete intersection singularities, then $Z$ is a $\mathbf{P}^{2}$-bundle over $C_{R}$ by a theorem of Badescu ([1, Theorem 5.5.3]); in the general case we need to work more. Using $\pi^{\prime}$ we see that $C_{R}=C_{R^{\prime}}$ for any $R, R^{\prime}$ and that in this way we define a fibration $\pi_{\mathrm{reg}}: X_{\mathrm{reg}} \rightarrow C_{R}$ such that $\pi_{\text {reg }} / U=\pi^{\prime}$. Set $C:=C_{R}$. Varying the hyperplane $R$ we obtain that for every plane $V \in \mathbf{T}$ we have $\operatorname{Card}(V \backslash V \cap \operatorname{Sing}(X))=1$. Let $\Gamma \subset X \times G(3, N+1)$ be the closure of the restriction to the fibers of $\pi^{\prime}$ of the incidence correspondence and let $\Phi$ be the normalization of $\Gamma$. Since $\operatorname{Card}(V \backslash V \cap \operatorname{Sing}(X))=1$ for every plane 
$V \in \mathbf{T}$, we obtain a morphism $\pi^{\prime \prime}: \Phi \rightarrow C$. The projection $\Phi \rightarrow X$ factors through a birational morphism $\alpha: \Phi \rightarrow Z$. Since $\operatorname{dim}(X)=3$ and $X$ is not a cone, for every $Q \in \operatorname{Sing}(X)$ there are only finitely many planes containing $Q$ and contained in $X$. Hence $\alpha$ is finite. Since $Z$ is normal, $\alpha$ is an isomorphism and hence $Z$ is a $\mathbf{P}^{2}$-bundle over the smooth curve $C$. In particular, $Z$ is smooth.

Proposition 8. Let $X \subset \mathbf{P}^{N}, N \geq 6$, be an integral variety with $\operatorname{dim}(X)=4$, $\operatorname{Sing}(X)$ finite and $\delta(N-1, X)>0$. Let $g: Z \rightarrow X$ be the normalization. Then either $X$ is a cone or $Z$ is a smooth scroll over a smooth curve, say $\pi: Z \rightarrow C$, and $g$ sends every fiber of $\pi$ isomorphically onto a 3-dimensional linear subspace contained in $X$.

ProOF. By $[2$, Corollary 3.3 (b)], we may assume $\operatorname{Sing}(X) \neq \emptyset$. By Remark 5 either $X$ is a cone or Condition (\$) is satisfied and in particular $\operatorname{dim}(X)-\delta(N-1, X)$ is even (Remark 6). Hence, $\delta(N-1, X)=2$. Fix $Q \in X$ and let $Z$ be a general hyperplane section of $X$ containing $Q$. By Bertini's theorem we have $\operatorname{Sing}(Z) \subseteq\{Q\}$. We have $\delta(N-2, Z) \geq \delta(N-1, X)-1=1$. Hence we may apply Proposition 7 to $Z$ and then apply the same proof taking $Z$ instead of $X \cap R$.

THEOREM 1. Assume char $(\mathbf{K}) \neq 2$. Let $X \subset \mathbf{P}^{N}$ be an irreducible normal reflexive $n$-dimensional variety with $\operatorname{Sing}(X) \neq \emptyset$ and $\delta(N-1, X)>0$. Assume $2 \delta(N-1, X) \geq n+\operatorname{dim}(\operatorname{Sing}(X))$. Then $\operatorname{Sing}(X)$ is a linear space and $X$ is a cone with $\operatorname{Sing}(X)$ as its vertex.

PROOF. Set $k:=\delta(N-1, X)$. Using Remark 3 and the preservation of reflexivity for general hyperplane sections ([10, Theorem 22 (i)], or [6, 5.9 and 5.12]), we reduce the general case to the case in which $\operatorname{Sing}(X)$ is finite; it is quite subtle (but true in arbitrary characteristic) that if a general hyperplane section of $X$ is a cone, then $X$ is a cone (see the second part titled 'When is the general hyperplane section of a variety a cone?' of [7]). In order to obtain a contradiction we assume that $X$ is not a cone. Since $\operatorname{Sing}(X)$ is finite and $X$ is not a cone we may assume Condition (\$) (Proposition 2). By Remark 6 and Proposition 6 we may assume $n \geq 5$. Fix a general $P \in X$ and a general contact locus $L_{0}$ with $P \in L_{0}$. We will follow the proof of [2, Theorem 4.2]. Let $F(P)$ (or just $Q_{0}$ as in [2, page 903]) be the connected component containing $L_{0}$ of the set of all $k$-dimensional linear spaces in $X$ which are deformations of $L_{0}$ and contain $P$; parts (a), (b) and (c) of [2, Lemma 4.2] work verbatim by Condition (\$) because these parts concern only a general element of $F(P)$; part (d) of [2, Lemma 4.2] works for the elements $L \in F(P)$ with $l \cap \operatorname{Sing}(X)=\emptyset$; since $\operatorname{Sing}(X)$ is finite, part (d) of [2, Lemma 4.2] is true by Proposition 2. Thus we may obtain [2, Lemma 4.3], that is, the existence of a linear space $D_{0} \subset X$ with $L_{0} \subset D_{0}$ and $\operatorname{dim}\left(D_{0}\right)=(n+k) / 2 ; D_{0}$ is the union of all $k$-planes in the family 
$F(P)$. Since $D_{0}$ depends only on $P$ (assumed to be general), we will set $D(P):=D_{0}$. Then we conclude as in the proof of Proposition 7.

If $\operatorname{char}(\mathbf{K})=0$ and $Z$ is assumed to have only locally complete intersection singularities, Theorem 1 follows from a theorem of Sommese ([1, Theorem 5.5.2]).

\section{Zak's Tangency Theorem}

In this section we discuss the existence of positive dimensional fibers of the ordinary Gauss map $\gamma_{n, X}$. Of course, by Zak's Tangency Theorem ([16, Theorem 1.7]) the variety $X$ cannot be smooth.

REMARK 7. Let $X \subset \mathbf{P}^{N}$ be an integral $n$-dimensional variety, $P \in X$ and let $V \subset \mathbf{P}^{N}$ be a linear space with $\operatorname{dim}(V)=n$ which is $J$-tangent to $X$ at $P$ in the sense of [16, Definition 1.6]. Then $X$ must be smooth at $P$ and $V=T_{P} X$. This trivial observation shows why in the case of the ordinary Gauss map Theorem 1.7 of [16] covers only the case in which the contact locus is contained in $X_{\text {reg }}$. This observation was one of the motivations for this paper.

EXAMPLE 4. Here we make no restriction on char(K). Fix homogeneous coordinates $x_{0}, \ldots, x_{3}$ of $\mathbf{P}^{3}$ and set $H:=\left\{x_{0}=0\right\}$. Fix an integer $s \geq 1$ and integers $a_{1}, \ldots, a_{s}, m_{1}, \ldots, m_{s}$ with $a_{i} \geq 1$ and $m_{i} \geq 2$ for every $i$. Fix an integer $d \geq \sum_{i=1}^{s} a_{i} m_{i}$. In the plane $H$ we fix $s$ distinct integral curves $R_{1}, \ldots, R_{s}$ with $\operatorname{deg}\left(R_{i}\right)=a_{i}$. Set $R:=\bigcup_{i=1}^{s} R_{i}$. We want to find a degree $d$ normal surface $A \subset \mathbf{P}^{3}$ such that $H$ is tangent to $A$ at each point of $R_{\text {reg }}$ and such that the scheme $A \cap H$ contains each curve $R_{i}$ with multiplicity $m_{i}$. Let $B$ be any reduced curve contained in $H$ with $\operatorname{deg}(B)=d-\sum_{i=1}^{s} a_{i} m_{i}$ and $\operatorname{card}(B \cap R)$ finite; if $d=\sum_{i=1}^{s} a_{i} m_{i}$ we take $B=\emptyset$. Set $C:=B \cup\left(\bigcup_{i=1}^{s} m_{i} R_{i}\right)$. Hence $C$ is a degree $d$ plane curve. We will show that we may find such a normal surface $A$ with $H \cap A$ containing $C$ (as schemes), $A$ smooth at every point not on $H$ and smooth at every point of $B \cup \operatorname{Sing}(R)$. Let $W$ be the linear system of all degree $d$ surfaces in $\mathbf{P}^{3}$ containing $C$ and hence either containing $H$ or with $C$ as a scheme-theoretic intersection with $H$. By the definition of $W$ and Bezout theorem every $A^{\prime} \in W$ not containing $H$ is smooth at each point of $B_{\text {reg. }}$. Taking reducible surfaces $H \cup F^{\prime} \in W$ with $F^{\prime}$ as any degree $d-1$ surface we see that the linear system $W$ has no base point outside $C$ and separates the tangent vectors at each point of $P^{3} \backslash H$. Hence by Bertini's theorem ([8, Theorem 6.3, part 4]) general $F \in W$ is smooth outside $H$. Since $\operatorname{Sing}(R) \cup \operatorname{Sing}(B)$ is finite, we easily see that general $A^{\prime} \in W$ is smooth at each point of $\operatorname{Sing}(R) \cup \operatorname{Sing}(B)$. Let $X=\{f=0\}$ be an irreducible degree $d$ surface containing $C$. Since $H=\left\{x_{0}=0\right\}$ and $X$ contains every irreducible component of $R$ with multiplicity at least 2, the Euler sequence of 
$T \mathbf{P}^{3}$ shows that $\operatorname{Sing}(X) \cap R=H \cap\left\{\partial f / \partial x_{0}\right\}$. Hence we see that such a general $X$ has exactly $(d-1)\left(\sum_{i=1}^{s} a_{i}\right)$ singular points, each of them on $R_{\text {reg }}$, exactly $(d-1) a_{i}$ of them on $R_{i}, 1 \leq i \leq s$, and that each of these singular points is an ordinary double point. However, taking particular equations $f$ we may find $X$ with a smaller number of singular points, although these singular points may be non-ordinary or with higher multiplicity. For instance, take $s=1$ and $R$ smooth. Fix a degree $d-1$ homogeneous polynomial $g\left(x_{1}, x_{2}, x_{3}\right)$ in 3 variables such that $w:=\operatorname{card}\left(R \cap\left\{g\left(x_{1}, x_{2}, x_{3}\right)=0\right\}\right)$ is as small as possible. For $\operatorname{deg}(R) \leq 3$, we may take $w=1$. There exists a degree $d$ polynomial $f$ with $\{f=0\} \cap H=C$ and with $\partial f / \partial x_{0} \equiv g\left(x_{1}, x_{2}, x_{3}\right) \bmod \left(x_{0}\right)$. At least in some cases (for instance when $R$ is a line) for the general polynomial $f$ with these properties the surface $\{f=0\}$ is smooth outside $R$.

Now we will show that, at least for non-normal varieties, we cannot extend Zak's Tangency Theorem making assumptions on their birational model, for instance to be very ample.

EXAMPLE 5. Fix an integer $n \geq 2$, a smooth $n$-dimensional variety $Z$ and an effective Cartier divisor $C \subset Z$. Let $D$ be an effective divisor such that the line bundle $\mathscr{O}_{Z}(2 C+D)$ is very ample. Consider the complete embedding $\phi$ of $Z$ into $\mathbf{P}^{s}:=\mathbf{P}\left(H^{0}\left(Z, \mathscr{O}_{Z}(2 C+D)\right)\right)$ and let $H$ be the hyperplane of $\mathbf{P}^{s}$ corresponding to the divisor $2 C+D$. By construction $H$ is tangent to $\phi(Z)$ along $\phi(C)$. Assume that for a general linear subspace $M$ of $H$ with $\operatorname{dim}(M)=s-n-2$, the linear projection of $\mathbf{P}^{s}$ from $M$ into $\mathbf{P}^{n+1}$ induces a birational map of $\phi(Z)$ and of $C$. Let $X$ and $C^{\prime}$ be the corresponding images. Assume that $X$ is not singular at the general point of every irreducible component of $C^{\prime}$. Let $\Pi$ be the hyperplane of $\mathbf{P}^{n+1}$ image of $H$ through the projection from $M$. By construction $\Pi$ is tangent to $X$ along $C^{\prime}$ and $X$ is birational to $Z$.

\section{References}

[1] M. Beltrametti and A. J. Sommese, The adjunction theory of complex projective varieties (Walter de Gruyter, Berlin, 1995).

[2] L. Ein, 'Varieties with small dual varieties, II', Duke Math. J. 52 (1985), 895-907.

[3] —_, 'Varieties with small dual varieties, I', Invent. Math. 86 (1986), 63-74.

[4] T. Fujita, 'Remarks on quasi-polarized varieties', Nagoya Math. J. 115 (1989), 105-123.

[5] I. M. Gelfand, M. M. Kapranov and A. V. Zelevinsky, Discriminants, resultants and multidimensional determinants (Birkhäuser, Boston, 1994).

[6] A. Hefez and S. L. Kleiman, 'Notes on duality for projective varieties', in: Geometry today (eds. E. Arbarello, C. Procesi and E. Strickland), Progr. Math. 60 (Birkhäuser, Boston, 1985) pp. 143-185.

[7] M. L. Johnson, Higher secant varieties (Ph.D. Thesis, Brandeis University, 1994). 
[8] J.-P. Jouanolou, Théorèmes de Bertini et applications, Progr. Math. 42 (Birkhäuser, Boston, 1983).

[9] H. Kaji, 'On the inseparable degrees of the Gauss map and the projection of the conormal variety to the dual of higher order for space curves', Math. Ann. 292 (1992), 529-532.

[10] S. L. Kleiman, 'The enumerative theory of singularities', in: Real and Complex Singularities, Oslo, 1976 (ed. P. Holm) (Sijitoff and Noordhoof, Alphen aan den Rijn, 1977) pp. 297-396.

[11] _ 'Tangency and duality', in: Proc. 1984 Vancouver Conference in Algebraic Geometry, CMS-AMS Conference Proceedings 6 (Amer. Math. Soc., Providence, RI, 1986).

[12] S. L. Kleiman and R. Piene, 'On the inseparability of the Gauss map', in: Enumerative Algebraic Geometry, Proc. Zeuthen Symposium, Contemp. Math. 123 (Amer. Math. Soc., Providence, RI, 1991) pp. 149-160.

[13] J. Landsberg, 'Algebraic geometry and projective differential geometry-Seoul National University concentrated lecture series', preprint math.AG/9809184, 1997.

[14] H. Tango, 'On $(n-1)$-dimensional projective spaces contained in the Grassmann variety $\mathrm{Gr}(n, 1)$ ', J. Math. Kyoto Univ. 14 (1974), 415-460.

[15] —_, 'On morphisms from projective space $\mathbf{P}^{n}$ ', J. Math. Kyoto Univ. 16 (1976), 201-207.

[16] F. L. Zak, Tangents and secants of algebraic varieties, Transl. Math. Monographs 127 (Amer. Math. Soc., Providence, RI, 1983).

Department of Mathematics

University of Trento

38050 Povo (TN)

Italy

e-mail: ballico@science.unitn.it 